\title{
An elementary operator and generalized Weyl's theorem
}

\author{
Fugen Gao* and Xiaochun Li
}

\section{"Correspondence:}

gaofugen08@126.com

College of Mathematics and

Information Science, Henan Normal

University, Xinxiang, Henan 453007,

China

\begin{abstract}
A Hilbert space operator $T$ belongs to class $A$ if $\left|T^{2}\right|-|T|^{2} \geq 0$. Let $d_{A B}$ denote either $\delta_{A B}$ or $\triangle_{A B}$, where $\delta_{A B}$ and $\triangle_{A B}$ denote the generalized derivation and the elementary operator on a Banach space $B(\mathcal{H})$ defined by $\delta_{A B} X=A X-X B$ and $\triangle_{A B} X=A X B-X$ respectively. If $A$ and $B^{*}$ are class $A$ operators, we show that $d_{A B}$ is polaroid and generalized Weyl's theorem holds for $f\left(d_{A B}\right)$, generalized $a$-Weyl's theorem holds for $f\left(\left(d_{A B}\right)^{*}\right)$ for every $f \in H\left(\sigma\left(d_{A B}\right)\right)$ and $f$ is not constant on each connected component of the open set $U$ containing $\sigma\left(d_{A B}\right)$, where $H\left(\sigma\left(d_{A B}\right)\right)$ denotes the set of all analytic functions in a neighborhood of $\sigma\left(d_{A B}\right)$.
\end{abstract}

MSC: 47B20; 47A63

Keywords: class A operators; generalized derivation; elementary operator; generalized Weyl's theorem; generalized a-Weyl's theorem

\section{Introduction}

Let $\mathcal{H}$ be a complex Hilbert space and $\mathbb{C}$ be the set of complex numbers. Let $B(\mathcal{H})$ and $K(\mathcal{H})$ denote the $C^{*}$-algebra of all bounded linear operators and the ideal of compact operators acting on $\mathcal{H}$ respectively. For operators $A, B \in B(\mathcal{H})$, let $\delta_{A B} \in B(B(\mathcal{H}))$ denote the generalized derivation on a Banach space $B(\mathcal{H})$ defined by $\delta_{A B} X=A X-X B$; let $\triangle_{A B} \in B(B(\mathcal{H})$ ) denote the elementary operator on a Banach space $B(\mathcal{H})$ defined by $\triangle_{A B}=A X B-X$. Let $d_{A B}$ denote either $\delta_{A B}$ or $\triangle_{A B}$. $d_{A B}$ has been studied by a number of authors [1-4]. Also let $\alpha(T)=\operatorname{dim} \operatorname{ker} T, \beta(T)=\operatorname{dim} \operatorname{ker} T^{*}$, and let $\sigma(T), \sigma_{a}(T)$ denote the spectrum and approximate point spectrum of $T$. An operator $T \in B(\mathcal{H})$ is called upper (resp. lower) semi-Fredholm if $\operatorname{ran} T$ is closed and $\alpha(T)<\infty$ (resp. $\beta(T)<\infty)$. In the sequel, let $S F_{+}(\mathcal{H})$ denote the set of all upper semi-Fredholm operators. If both $\alpha(T)$ and $\beta(T)$ are finite, then $T$ is called a Fredholm operator. An operator $T \in B(\mathcal{H})$ is called Weyl if it is Fredholm of index zero and Browder if it is Fredholm of finite ascent and descent. Let $\sigma_{e}(T), \sigma_{w}(T)$ and $\sigma_{b}(T)$ denote the essential spectrum, the Weyl spectrum and the Browder spectrum of $T \in B(\mathcal{H})$. Let iso $\mathcal{K}$ denote the isolated points of $\mathcal{K} \subseteq \mathbb{C}$. We write $\pi_{00}(T)=\{\lambda \in$ iso $\sigma(T): 0<\alpha(T-\lambda)<\infty\}, \pi_{00}^{a}(T)=\left\{\lambda \in\right.$ iso $\left.\sigma_{a}(T): 0<\alpha(T-\lambda)<\infty\right\}$, and $p_{00}(T)=\sigma(T) \backslash \sigma_{b}(T)$. It is evident that $\sigma_{e}(T) \subseteq \sigma_{w}(T) \subseteq \sigma_{b}(T)=\sigma_{e}(T) \bigcup \operatorname{acc} \sigma(T)$ and $p_{00}(T) \subseteq \pi_{00}(T) \subseteq \pi_{00}^{a}(T)$, where acc $\sigma(T)=\sigma(T) \backslash$ iso $\sigma(T)$.

We say that Weyl's theorem holds for $T \in B(\mathcal{H})$ if

$$
\sigma(T) \backslash \sigma_{w}(T)=\pi_{00}(T),
$$

( 2012 Gao and Li; licensee Springer. This is an Open Access article distributed under the terms of the Creative Commons Attribution License (http://creativecommons.org/licenses/by/2.0), which permits unrestricted use, distribution, and reproduction in any medium, provided the original work is properly cited. 
and that Browder's theorem holds for $T \in B(\mathcal{H})$ if

$$
\sigma(T) \backslash \sigma_{w}(T)=p_{00}(T)
$$

By definition, $\sigma_{e a}(T)=\bigcap\left\{\sigma_{a}(T+K): K \in K(\mathcal{H})\right\}$ is the essential approximate point spectrum of $T$, and $\sigma_{a b}(T)=\bigcap\left\{\sigma_{a}(T+K): K \in K(\mathcal{H})\right.$ and $\left.K T=T K\right\}$ is the Browder approximate point spectrum of $T$.

We say that $a$-Weyl's theorem holds for $T \in B(\mathcal{H})$ if

$$
\sigma_{a}(T) \backslash \sigma_{e a}(T)=\pi_{00}^{a}(T),
$$

and that $a$-Browder's theorem holds for $T \in B(\mathcal{H})$ if

$$
\sigma_{e a}(T)=\sigma_{a b}(T)
$$

For a bounded linear operator $T$ and a nonnegative integer $n$, define $T_{n}$ to be the restriction of $T$ to ran $T^{n}$ viewed as a map from $\operatorname{ran} T^{n}$ into $\operatorname{ran} T^{n}$ (in particular $T_{0}=T$ ). If for some integer $n$, the range space $\operatorname{ran} T^{n}$ is closed and $T_{n}$ is a Fredholm operator, then $T$ is called a B-Fredholm operator. If $T$ is a B-Fredholm operator of index zero, then $T$ is called a B-Weyl operator. The B-Fredholm spectrum $\sigma_{\mathrm{BF}}(T)$ and B-Weyl spectrum $\sigma_{\mathrm{BW}}(T)$ of $T$ are defined by $\sigma_{\mathrm{BF}}(T)=\{\lambda \in \mathbb{C}: T-\lambda$ is not a B-Fredholm operator $\}$ and $\sigma_{\mathrm{BW}}(T)=\{\lambda \in \mathbb{C}: T-\lambda$ is not a B-Weyl operator $\}$. An operator $T \in B(\mathcal{H})$ satisfies generalized Weyl's theorem [5, Definition 2.13] if

$$
\sigma_{\mathrm{BW}}(T)=\sigma(T) \backslash \pi_{0}(T),
$$

where $\pi_{0}(T)$ is the set of all isolated eigenvalues of $T$, and $T \in B(\mathcal{H})$ satisfies generalized Browder's theorem [5, Definition 2.13] if

$$
\sigma_{\mathrm{BW}}(T)=\sigma(T) \backslash p_{0}(T),
$$

where $p_{0}(T)$ is the set of all poles of the resolvent of $T$.

Let $\operatorname{SBF}_{+}(\mathcal{H})$ be the class of all the upper semi-B-Fredholm operators and $\operatorname{SBF}_{+}^{-}(\mathcal{H})$ be the class of all $T \in \operatorname{SBF}_{+}(\mathcal{H})$ such that $\operatorname{ind}(T) \leq 0$. Let

$$
\sigma_{\mathrm{SBF}_{+}^{-}}(T)=\left\{\lambda \in \mathbb{C}: T-\lambda \notin \operatorname{SBF}_{+}^{-}(\mathcal{H})\right\}
$$

be called the semi-B-essential approximate point spectrum of $T$. We say that $T \in B(\mathcal{H})$ satisfies generalized $a$-Weyl's theorem [5, Definition 2.13] if

$$
\sigma_{\mathrm{SBF}_{+}^{-}}(T)=\sigma_{a}(T) \backslash \pi_{0}^{a}(T),
$$

where $\pi_{0}^{a}(T)$ is the set of all eigenvalues of $T$ which are isolated points of $\sigma_{a}(T)$. 
The following implications are known to hold:

$$
\begin{aligned}
& \text { generalized } a \text {-Weyl's theorem } \\
& \Rightarrow \text { generalized Weyl's theorem } \Longrightarrow \text { Weyl's theorem } \\
& \Rightarrow \quad \text { Browder's theorem; }
\end{aligned}
$$

generalized $a$-Weyl's theorem

$\Longrightarrow a$-Weyl's theorem $\quad \Longrightarrow \quad a$-Browder's theorem

$\Longrightarrow$ Browder's theorem.

In this paper, we shall study the generalized Weyl's theorem for the elementary operator and the generalized derivation with class A operators as entries. Recall that $T \in B(\mathcal{H})$ is called $p$-hyponormal for $p>0$ if $\left(T^{*} T\right)^{p}-\left(T T^{*}\right)^{p} \geq 0$ [6]; when $p=1, T$ is called hyponormal. And $T$ is called paranormal if $\|T x\|^{2} \leq\left\|T^{2} x\right\|\|x\|$ for all $x \in \mathscr{H}[7,8]$. In order to discuss the relations between paranormal and $p$-hyponormal and log-hyponormal operators ( $T$ is invertible and $\log T^{*} T \geq \log T T^{*}$ ), Furuta, Ito and Yamazaki [9] introduced a very interesting class of operators: class A defined by $\left|T^{2}\right|-|T|^{2} \geq 0$, where $|T|=\left(T^{*} T\right)^{\frac{1}{2}}$ which is called the absolute value of $T$, and they showed that class $\mathrm{A}$ is a subclass of paranormals and contains $p$-hyponormal and log-hyponormal operators.

Definition 1.1 An operator $T \in B(\mathcal{H})$ is said to have the single valued extension property (SVEP) at $\lambda \in \mathbb{C}$ if for every open neighborhood $\mathcal{G}$ of $\lambda$, the only function $f \in H(\mathcal{G})$ such that $(T-\mu) f(\mu)=0$ on $G$ is $0 \in H(\mathcal{G})$, where $H(\mathcal{G})$ means the space of all analytic functions on $G$. When $T$ has SVEP at each $\lambda \in \mathbb{C}$, say that $T$ has SVEP.

The single valued extension property dates back to the early days of local spectral theory; see the recent monograph of Laursen and Neumann [10] or Aiena [11]. In addition to the definition of SVEP, there are notions of property $(\beta)$, property $(\delta)$ and condition $(C)$. The interested reader is referred to [10] for more details.

\section{The main results}

For $T \in B(\mathcal{H})$, let $L_{T}$ and $R_{T}$ denote the operators of left and right multiplication by $T$ respectively.

Chō and Yamazaki proved that class A operators have property $\beta$ in [12] Theorem 3.1; unfortunately, there are some mistakes in the proof of this theorem; see details in [13]. So, Theorem 3.1 in [12] is still an open problem.

Lemma 2.1 Let $A$ and $B^{*}$ be class $A$ operators satisfying property $(\beta)$, then $d_{A B}$ has SVEP.

Proof By assumption and [10] Theorem 2.5.5, $A$ satisfies property $(\beta)$ and $B$ satisfies property $(\delta)$. Hence, both $L_{A}$ and $R_{B}$ satisfy condition $(C)$ by [10] Corollary 3.6.11. Clearly, $L_{A}$ and $R_{B}$ commute. By Theorem 3.6.3 and Note 3.6.19 on p.283 of [10], $L_{A}-R_{B}$ and $L_{A} R_{B}$ have SVEP, which implies that $d_{A B}$ has SVEP.

It is well known that the isolated points of the spectrum of a class A (indeed, paranormal) operator $T$ are poles of the resolvent of the operator (hence, eigenvalues of the operator), 
the restriction of $T$ to an invariant subspace is again of class A (resp., paranormal), and that if $T$ has countable spectrum then $T$ is normal. (We shall use this information freely in the following without any further reference.)

Recall, [14], that $\sigma\left(\delta_{A B}\right)=\{\lambda: \lambda \in \sigma(A)-\sigma(B)\}$ and $\sigma\left(\triangle_{A B}\right)=\{\lambda: \lambda \in \sigma(A) \sigma(B)-1\}$. If $\lambda \in$ iso $\sigma\left(d_{A B}\right)$, then we have one of the following two cases:

(1) $\lambda \neq-1$ if $d_{A B}=\triangle_{A B}$. Then there exist finite sequences $\left\{\alpha_{i}\right\}_{i=1}^{m}$ and $\left\{\beta_{i}\right\}_{i=1}^{m}$, where $\alpha_{i} \in$ iso $\sigma(A)$ and $\beta_{i} \in$ iso $\sigma(B)$ respectively, such that $\lambda=\alpha_{i}-\beta_{i}$ if $\lambda \in$ iso $\sigma\left(\delta_{A B}\right)$ and $\lambda=\alpha_{i} \beta_{i}-1$ if $\lambda \in$ iso $\sigma\left(\triangle_{A B}\right)$, for all $1 \leq i \leq m$.

(2) $\lambda=-1$ and $d_{A B}=\triangle_{A B}$. Then either $0 \in$ iso $\sigma(A)$ and $0 \in$ iso $\sigma(B)$ or $0 \in$ iso $\sigma(A)$ and $0 \notin \sigma(B)$ or $0 \in$ iso $\sigma(B)$ and $0 \notin \sigma(A)$.

Theorem 2.2 Let $A$ and $B^{*}$ be class $A$ operators, then $H_{0}\left(d_{A B}-\lambda\right)=\operatorname{ker}\left(d_{A B}-\lambda\right)$ for all $\lambda \in \operatorname{iso} \sigma\left(d_{A B}\right)$.

Proof We consider the case $d_{A B}=\delta_{A B}$ and $d_{A B}=\triangle_{A B}$ respectively.

(1) We consider the case $d_{A B}=\delta_{A B}$. The idea comes from [1]. If $\lambda \in \operatorname{iso} \sigma\left(\delta_{A B}\right)$, then there exist finite sets $\left\{\alpha_{1}, \alpha_{2}, \ldots, \alpha_{m}\right\}$ and $\left\{\beta_{1}, \beta_{2}, \ldots, \beta_{m}\right\}$, where $\alpha_{i} \in$ iso $\sigma(A)$ and $\beta_{i} \in$ iso $\sigma(B)$ such that $\lambda=\alpha_{i}-\beta_{i}$ for all $1 \leq i \leq m$. Let

$$
M_{1}=\bigvee_{i=1}^{m} \operatorname{ker}\left(A-\alpha_{i}\right), \quad M_{2}=\mathcal{H} \ominus M_{1}
$$

and

$$
N_{1}=\bigvee_{i=1}^{m} \operatorname{ker}\left(B^{*}-\overline{\beta_{i}}\right), \quad N_{2}=\mathcal{H} \ominus N_{1}
$$

Then $A$ and $B$ have representations $A=\left(\begin{array}{cc}A_{11} & A_{12} \\ 0 & A_{22}\end{array}\right)$ on $M_{1} \oplus M_{2}$ and $B=\left(\begin{array}{cc}B_{11} & 0 \\ B_{21} & B_{22} 2\end{array}\right)$ on $N_{1} \oplus N_{2}$ respectively, where $A_{11}$ and $B_{11}$ are normal, $\sigma(A)=\sigma\left(A_{11}\right) \cup \sigma\left(A_{22}\right), \sigma(B)=\sigma\left(B_{11}\right) \cup \sigma\left(B_{22}\right)$ and $\lambda \notin \sigma\left(\delta_{A_{i i} B_{i j}}\right)$ for all $1 \leq i, j \leq 2$ other than $i=j=1$. Consider an $X \in H_{0}\left(\delta_{A B}-\lambda\right)$. Letting $X: N_{1} \oplus N_{2} \longrightarrow M_{1} \oplus M_{2}$ have the matrix representation $X=\left[X_{i j}\right]_{i, j=1}^{2}$, we have

$$
\left(\delta_{A B}-\lambda\right)^{n} X=\left(\begin{array}{cc}
* & * \\
* & \left(\delta_{A_{22} B_{22}}-\lambda\right)^{n} X_{22}
\end{array}\right)
$$

for some yet to be determined entries $*$.

Since

$$
\lim _{n \rightarrow \infty}\left\|\left(\delta_{A B}-\lambda\right)^{n} X\right\|^{\frac{1}{n}}=0
$$

we have that

$$
\lim _{n \rightarrow \infty}\left\|\left(\delta_{A_{22} B_{22}}-\lambda\right)^{n} X_{22}\right\|^{\frac{1}{n}}=0 .
$$

Since $\lambda \notin \sigma\left(\delta_{A_{22} B_{22}}\right)$, we have that $\delta_{A_{22} B_{22}}-\lambda$ is invertible. Hence, $X_{22}=0$. So, we have that

$$
\left(\delta_{A B}-\lambda\right)^{n} X=\left(\begin{array}{cc}
* & \left(\delta_{A_{11} B_{22}}-\lambda\right)^{n} X_{12} \\
\left(\delta_{A_{22} B_{11}}-\lambda\right)^{n} X_{21} & 0
\end{array}\right) .
$$


Since

$$
\lim _{n \rightarrow \infty}\left\|\left(\delta_{A_{i i} B_{j j}}-\lambda\right)^{n} X_{i j}\right\|^{\frac{1}{n}}=0
$$

for all $1 \leq i, j \leq 2$ other than $i=j=1$ and since $\lambda \notin \sigma\left(\delta_{A_{11} B_{22}}\right)$ and $\lambda \notin \sigma\left(\delta_{A_{22} B_{11}}\right)$, we have that $X_{12}=X_{21}=0$. Hence,

$$
\left(\delta_{A B}-\lambda\right)^{n} X=\left(\begin{array}{cc}
\left(\delta_{A_{11} B_{11}}-\lambda\right)^{n} X_{11} & 0 \\
0 & 0
\end{array}\right) .
$$

Since $A_{11}$ and $B_{11}$ are normal,

$$
\lim _{n \rightarrow \infty}\left\|\left(\delta_{A_{11} B_{11}}-\lambda\right)^{n} X_{11}\right\|^{\frac{1}{n}}=0
$$

if and only if

$$
\left(\delta_{A_{11} B_{11}}-\lambda\right) X_{11}=0
$$

by [15] Lemma 2 . Hence, we have $H_{0}\left(\delta_{A B}-\lambda\right) \subseteq \operatorname{ker}\left(\delta_{A B}-\lambda\right)$. Since $\operatorname{ker}\left(\delta_{A B}-\lambda\right) \subseteq H_{0}\left(\delta_{A B}-\right.$ $\lambda$ ) is always true, we have

$$
H_{0}\left(\delta_{A B}-\lambda\right)=\operatorname{ker}\left(\delta_{A B}-\lambda\right) .
$$

(2) We consider the case $d_{A B}=\triangle_{A B}$. When $\lambda \neq-1$, the proof is similar to the proof of the first part. We omit the proof. When $\lambda=-1$, then either $0 \in$ iso $\sigma(A)$ and $0 \in$ iso $\sigma(B)$ or $0 \in$ iso $\sigma(A)$ and $0 \notin \sigma(B)$ or $0 \in$ iso $\sigma(B)$ and $0 \notin \sigma(A)$. If 0 is both in iso $\sigma(A)$ and iso $\sigma(B)$, then let $M_{1}=\operatorname{ker}(A), M_{2}=\mathcal{H} \ominus M_{1}$ and $N_{1}=\operatorname{ker}\left(B^{*}\right), N_{2}=\mathcal{H} \ominus N_{1}$.

Then we have $A=\left(\begin{array}{ll}0 & C_{1} \\ 0 & A_{2}\end{array}\right)$ on $\mathcal{H}=M_{1} \oplus M_{2}$ and $B=\left(\begin{array}{cc}0 & 0 \\ C_{2} & B_{2}\end{array}\right)$ on $\mathcal{H}=N_{1} \oplus N_{2}$ for some operators $C_{1}, A_{2}$ and $C_{2}, B_{2}$ respectively. Here both $A_{2}$ and $B_{2}$ are invertible. So, we have that $\triangle_{A_{2} B_{2}}-\lambda=L_{A_{2}} R_{B_{2}}$ is invertible. Let $X: N_{1} \oplus N_{2} \rightarrow M_{1} \oplus M_{2}$ have the matrix representation $X=\left[X_{i j}\right]_{i, j=1}^{2}$. If $X \in H_{0}\left(\triangle_{A B}-\lambda\right)=H_{0}\left(L_{A} R_{B}\right)$, it follows that $X_{22}=0$ as in the proof of the first part. Hence, $L_{A} R_{B} X=0$ for every $X \in H_{0}\left(\triangle_{A B}-\lambda\right)=H_{0}\left(L_{A} R_{B}\right)$. So, we have $H_{0}\left(\triangle_{A B}-\lambda\right) \subseteq \operatorname{ker}\left(\triangle_{A B}-\lambda\right)$. Since $\operatorname{ker}\left(\triangle_{A B}-\lambda\right) \subseteq H_{0}\left(\triangle_{A B}-\lambda\right)$ is always true, we have $H_{0}\left(\triangle_{A B}-\lambda\right)=\operatorname{ker}\left(\triangle_{A B}-\lambda\right)$. The proofs of the other remaining cases are similar, we consider $0 \in$ iso $\sigma(A)$ and $0 \notin \sigma(B)$. Here $\triangle_{A B}-\lambda=L_{A} R_{B}$. In the following, we shall prove that $H_{0}\left(L_{A} R_{B}\right)=H_{0}\left(L_{A}\right)$. If $X \in H_{0}\left(L_{A} R_{B}\right)$, then

$$
\lim _{n \rightarrow \infty}\left\|\left(L_{A}\right)^{n} X\right\|^{\frac{1}{n}}=\lim _{n \rightarrow \infty}\left\|\left(L_{A} R_{B}\right)^{n} X B^{-n}\right\|^{\frac{1}{n}} \leq\left\|B^{-1}\right\| \lim _{n \rightarrow \infty}\left\|\left(L_{A} R_{B}\right)^{n} X\right\|^{\frac{1}{n}}=0 .
$$

On the other hand, if $X \in H_{0}\left(L_{A}\right)$, then

$$
\lim _{n \rightarrow \infty}\left\|\left(L_{A} R_{B}\right)^{n} X\right\|^{\frac{1}{n}} \leq\|B\| \lim _{n \rightarrow \infty}\left\|\left(L_{A}\right)^{n} X\right\|^{\frac{1}{n}}=0 .
$$

Hence, $H_{0}\left(L_{A} R_{B}\right)=H_{0}\left(L_{A}\right)$. Next, we shall prove that $H_{0}\left(L_{A}\right)=\operatorname{ker}\left(L_{A}\right)$. Let $M_{1}=\operatorname{ker} A$, $M_{2}=\mathcal{H} \ominus M_{1}$. We have $A=\left(\begin{array}{ll}0 & C_{1} \\ 0 & A_{2}\end{array}\right)$ on $\mathcal{H}=M_{1} \oplus M_{2}$, where $A_{2}$ is invertible. Let $X$ have the following matrix representation: $X=\left[X_{i j}\right]_{i, j=1}^{2}$ on $\mathcal{H}=M_{1} \oplus M_{2}$. If $X \in H_{0}\left(L_{A}\right)$, as in 
the proof above, we have that $X_{21}=X_{22}=0$. So, we have that $L_{A} X=0$. Hence, $H_{0}\left(L_{A}\right) \subseteq$ $\operatorname{ker}\left(L_{A}\right)$. Since $\operatorname{ker}\left(L_{A}\right) \subseteq H_{0}\left(L_{A}\right)$ is always true, we have that $H_{0}\left(L_{A}\right)=\operatorname{ker}\left(L_{A}\right)$. Since $B$ is invertible, we have $\operatorname{ker}\left(L_{A} R_{B}\right)=\operatorname{ker}\left(L_{A}\right)$. Therefore, we have that

$$
H_{0}\left(L_{A} R_{B}\right)=H_{0}\left(L_{A}\right)=\operatorname{ker}\left(L_{A}\right)=\operatorname{ker}\left(L_{A} R_{B}\right),
$$

hence

$$
H_{0}\left(L_{A} R_{B}\right)=\operatorname{ker}\left(L_{A} R_{B}\right)
$$

That is,

$$
H_{0}\left(\triangle_{A B}-\lambda\right)=\operatorname{ker}\left(\triangle_{A B}-\lambda\right) .
$$

This completes the proof.

An operator $T \in B(\mathcal{H})$ is said to be isoloid if every isolated point of $\sigma(T)$ is an eigenvalue of $T$ and polaroid if every isolated point of $\sigma(T)$ is a pole of the resolvent of $T$. In general, if $T$ is polaroid then it is isoloid.

Lemma 2.3 Let $A$ and $B^{*}$ be class $A$ operators, then $d_{A B}$ and $d_{A B}^{*}$ are polaroid. In particular, $d_{A B}$ and $d_{A B}^{*}$ are isoloid.

Proof We only need to prove that $d_{A B}$ is polaroid. Let $\mathcal{X}=B(\mathcal{H})$ and $\lambda \in$ iso $\sigma\left(d_{A B}\right)$. Then we have that $H_{0}\left(d_{A B}-\lambda\right)=\operatorname{ker}\left(d_{A B}-\lambda\right)$ by Theorem 2.2. Hence,

$$
\mathcal{X}=H_{0}\left(d_{A B}-\lambda\right) \oplus K\left(d_{A B}-\lambda\right)=\operatorname{ker}\left(d_{A B}-\lambda\right) \oplus K\left(d_{A B}-\lambda\right) .
$$

So, we have

$$
\left(d_{A B}-\lambda\right) \mathcal{X}=0 \oplus\left(d_{A B}-\lambda\right)\left(K\left(d_{A B}-\lambda\right)\right)=K\left(d_{A B}-\lambda\right) .
$$

Therefore,

$$
\mathcal{X}=\operatorname{ker}\left(d_{A B}-\lambda\right) \oplus\left(d_{A B}-\lambda\right) \mathcal{X}
$$

Thus, isolated points of $\sigma\left(d_{A B}\right)$ are simple poles of the resolvent of $d_{A B}$. Hence, $d_{A B}$ is polaroid. So, we have that $d_{A B}$ and $d_{A B}^{*}$ are polaroid. Since polaroid operators are always isoloid, we have that $d_{A B}$ and $d_{A B}^{*}$ are isoloid.

Theorem 2.4 Let $A$ and $B^{*}$ be class $A$ operators satisfying property $(\beta)$. Then generalized Weyl's theorem holds for $f\left(d_{A B}\right)$ for every $f \in H\left(\sigma\left(d_{A B}\right)\right)$ and $f$ is not constant on each connected component of the open set $U$ containing $\sigma\left(d_{A B}\right)$.

Proof Suppose that $A$ and $B^{*}$ are class A operators. By Lemma 2.1 and Lemma 2.3, we have that $d_{A B}$ has SVEP and $d_{A B}$ is polaroid. So, we have that generalized Weyl's theorem 
holds for $d_{A B}$ by [16, Theorem 3.10(ii)]. Since $d_{A B}$ has SVEP and $d_{A B}$ is isoloid, we have that generalized Weyl's theorem holds for $f\left(d_{A B}\right)$ for every $f \in H\left(\sigma\left(d_{A B}\right)\right)$ by [17, Theorem 2.2].

Corollary 2.5 Let $A$ and $B^{*}$ be class A operators satisfying property $(\beta)$. Then Weyl's theorem holds for $f\left(d_{A B}\right)$ for every $f \in H\left(\sigma\left(d_{A B}\right)\right)$ and $f$ is not constant on each connected component of the open set $U$ containing $\sigma\left(d_{A B}\right)$.

A bounded linear operator $T \in B(\mathcal{H})$ is called $a$-isoloid if every isolated point of $\sigma_{a}(T)$ is an eigenvalue of $T$. Note that every $a$-isoloid operator is isoloid and the converse is not true in general.

Lemma 2.6 Let $A$ and $B^{*}$ be class $A$ operators satisfying property $(\beta)$, then $d_{A B}^{*}$ is a-isoloid.

Proof Let $\lambda$ be an isolated point of $\sigma_{a}\left(d_{A B}^{*}\right)$. Suppose that $A$ and $B^{*}$ are class A operators satisfying property $(\beta)$. By Lemma 2.1 and Lemma 2.3, we have that $d_{A B}$ has SVEP and $d_{A B}^{*}$ is isoloid. Hence, $\sigma_{a}\left(d_{A B}^{*}\right)=\sigma\left(d_{A B}^{*}\right)$ by [18, Corollary 7]. We have that $\lambda$ is an isolated point of $\sigma\left(d_{A B}^{*}\right)$. Since $d_{A B}^{*}$ is isoloid, we have that $\lambda$ is an eigenvalue of $d_{A B}^{*}$. Hence, $d_{A B}^{*}$ is $a$-isoloid.

Theorem 2.7 Let $A$ and $B^{*}$ be class $A$ operators satisfying property $(\beta)$. Then generalized a-Weyl's theorem holds for $f\left(d_{A B}^{*}\right)$ for every $f \in H\left(\sigma\left(d_{A B}\right)\right)$, and $f$ is not constant on each connected component of the open set $U$ containing $\sigma\left(d_{A B}\right)$.

Proof Suppose that $A$ and $B^{*}$ are class A operators satisfying property ( $\beta$ ). By Lemma 2.1 and Lemma 2.3, we have that $d_{A B}$ has SVEP and $d_{A B}^{*}$ is polaroid. By Corollary 2.5, Weyl's theorem holds for $d_{A B}$. Hence, $d_{A B}^{*}$ satisfies Weyl's theorem by [19, Proposition 2.1]. Since $d_{A B}$ has SVEP and $d_{A B}^{*}$ is polaroid, generalized $a$-Weyl's theorem holds for $d_{A B}^{*}$ by [16, Theorem 3.10]. $T$ is $a$-isoloid by Lemma 2.6, hence generalized $a$-Weyl's theorem holds for $f(T)$ for every $f \in H(\sigma(T))$ by [17, Theorem 2.4].

Corollary 2.8 Let $A$ and $B^{*}$ be class $A$ operators satisfying property $(\beta)$. Then a-Weyl's theorem holds for $f\left(d_{A B}^{*}\right)$ for every $f \in H\left(\sigma\left(d_{A B}\right)\right)$, and $f$ is not constant on each connected component of the open set $U$ containing $\sigma\left(d_{A B}\right)$.

Competing interests

The authors declare that they have no competing interests.

Authors' contributions

All authors contributed equally to the writing of the present article. And they also read and approved the final manuscript.

Acknowledgements

The authors wish to express their indebtedness to the referee, for his suggestions have improved the final version of the present work. This work was supported by the National Natural Science Foundation of China (11071188), (11271112); the Natural Science Foundation of the Department of Education, Henan Province (2011A110009) and the Project of Science and Technology Department of Henan Province (122300410375), (112300410323). 


\section{References}

1. Chō, M, Djordjević, SV, Duggal, BP: Bishop's property $(\beta)$ and an elementary operator. Hokkaido Math. J. 40(3), 337-356 (2011)

2. Chō, M, Djordjević, SV, Duggal, BP, Yamazaki, T: On an elementary operator with $w$-hyponormal operator entries. Linear Algebra Appl. 433, 2070-2079 (2010)

3. Duggal, BP: An elementary operator with log-hyponormal, $p$-hyponormal entries. Linear Algebra Appl. 428 1109-1116 (2008)

4. Lombarkia, F: Generalized Weyl's theorem for an elementary operators. Bull. Math. Anal. Appl. 3, 123-131 (2011)

5. Berkani, M, Koliha, JJ: Weyl type theorems for bounded linear operators. Acta Sci. Math. (Szeged) 69, $359-376$ (2003)

6. Aluthge, A: On $p$-hyponormal operators for $0<p<1$. Integral Equ. Oper. Theory 13, 307-315 (1990)

7. Furuta, T: On the class of paranormal operators. Proc. Jpn. Acad. 43, 594-598 (1967)

8. Furuta, T: Invitation to Linear Operators. Taylor \& Francis, London (2001)

9. Furuta, T, Ito, M, Yamazaki, T: A subclass of paranormal operators including class of log-hyponormal and several classes. Sci. Math. 1(3), 389-403 (1998)

10. Laursen, KB, Neumann, MM: Introduction to Local Spectral Theory. Clarendon Press, Oxford (2000)

11. Aiena, P: Fredholm and Local Spectral Theory with Applications to Multipliers. Kluwer Academic, Dorchecht (2004)

12. Chō, M, Yamazaki, T: An operator transform from class A to the class of hyponormal operators and its application. Integral Equ. Oper. Theory 53, 497-508 (2005)

13. Chō, M, Yamazaki, T: Erratum to 'An operator transform from class A to the class of hyponormal operators and its application' [Integral Equations and Operator Theory, 53 497-508 (2005)] (to appear)

14. Embry, MR, Rosenblum, M: Spectra, tensor products, and linear operator equations. Pac. J. Math. 53, 95-107 (1974)

15. Radjabalipour, M: An extension of Putnam-Fuglede theorem for hyponormal operators. Math. Z. 194, 117-120 (1987)

16. Aiena, P, Aponte, E, Balzan, E: Weyl type theorems for left and right polaroid operators. Integral Equ. Oper. Theory 66, $1-20(2010)$

17. Zguitti, H: A note on generalized Weyl's theorem. J. Math. Anal. Appl. 316, 373-381 (2006)

18. Finch, JK: The single valued extension property on a Banach space. Pac. J. Math. 58, 61-69 (1975)

19. Duggal, BP: Polaroid operators satisfying Weyl's theorem. Linear Algebra Appl. 414, 271-277 (2006)

doi:10.1186/1029-242X-2012-243

Cite this article as: Gao and Li: An elementary operator and generalized Weyl's theorem. Journal of Inequalities and Applications 2012 2012:243.

\section{Submit your manuscript to a SpringerOpen ${ }^{\circ}$ journal and benefit from:}

- Convenient online submission

Rigorous peer review

- Immediate publication on acceptance

- Open access: articles freely available online

- High visibility within the field

- Retaining the copyright to your article 